\title{
TDR-based Multiple Leak Detection System using an S-parameter Transmission Line Model for Long-Distance Pipelines
}

\author{
Sihyeong Woo ${ }^{1}$, Taejin Kim², Junmin Lee ${ }^{3}$, Hyunseok $\mathrm{Oh}^{4}$, Byeng D. Youn ${ }^{* 5}$ and Daeil Kwon ${ }^{6}$ \\ ${ }^{1}$ Republic of Korea Air Force, Dunsan-ro, Dong-gu, Daegu, 41053, Republic of Korea \\ wsj1697@gmail.com \\ ${ }^{2,3,5}$ Department of Mechanical Engineering, Seoul National University, Gwanak-ro, Gwanak-gu, Seoul, 08826, Republic of \\ Korea \\ godori16@snu.ac.kr \\ 13.jmlee@gmail.com \\ ${ }^{4}$ School of Mechanical Engineering, Gwangju Institute of Science and Technology, Gwangju, 61005, Republic of Korea \\ hsoh@gist.ac.kr \\ ${ }^{6}$ School of Design and Human Engineering, Ulsan National Institute of Science and Technology, Ulsan, 08826, Republic of \\ Korea \\ dkwon@unist.ac.kr
}

\begin{abstract}
Leaks in water distribution systems should be detected to avoid economic, environmental, and social problems. Existing Bayesian Inference based time-domainreflectometry (TDR) methods for leak detection have a limitation for real applications due to the lengthy time in building sample data. As the pipeline distance becomes longer and multiple leaks must be considered in long distance pipelines, the computational time for building training data gets larger. This paper proposes a scattering-parameter-based forward model to relieve computational burden of the existing TDR methods. It was shown that the proposed model outperformed the existing RLGC-based forward model in terms of computational time. The proposed model that is combined with Bayesian inference and TDR signal modeling is validated with an experimental pipeline, leak detectors, transmission line, and TDR instrument for leak detection. In summary, the proposed method is promising for leak detection in long pipelines as well as multiple leaks.
\end{abstract}

\section{INTRODUCTION}

In the water distribution system, water is supplied from its source to users through a pipeline. During water transfer, often a large amount of water is not supplied to the end user

\footnotetext{
Sihyeong Woo et al. This is an open-access article distributed under the terms of the Creative Commons Attribution 3.0 United States License, which permits unrestricted use, distribution, and reproduction in any medium, provided the original author and source are credited.

*Corresponding author: Byeng D. Youn (e-mail: bdyoun@snu.ac.kr).
}

but instead leaks from the pipeline along the pipeline route. According to the International Water Supply Association (IWSA), 20-30 percent of total produced water is not supplied to users as a result of severalcauses including leaks (Hunaidi, Chu, Wang, \& Guan, 2000) (Cheong, 1991) (Thornton, Sturm, \& Kunkel, 2008) (Moe, \& Rheingans, 2006). For example, 250 billion liters of water annually leak from pipelines in the Great Lakes states; this quantity of water could serve the needs of 1.9 million Americans for a year (CNT, 2013). Unaddressed leaks not only waste resources and money but also cause environmental and social problems, such as sinkholes. Thus, it is very important to detect leaks to avoid these problems. However, this is not an easy task because most pipelines are buried underground or - in the case of long-distance pipelines - installed in remote regions. Numerous methods including leak noise correlators (Gao, Brennan, Joseph, Muggleton, \& Hunaidi, 2004), ground penetrating radar (O'Brien, Murray, \& McDonald, 2003) (Demirci, Yigit, Eskidemir, \& Ozdemir, 2012) (Costello, Chapman, Rogers, \& Metje 2007) and pigmounted acoustic sensing (McNulty, 2001) have been proposed to detect pipeline leaks. The methods are suitable for inspecting a specific area where leaks are suspected based on prior information, such as a civil complaint. To the best use of the methods, surveyors should be dispatched to the suspected leak area. The methods are most appropriate to detect leaks over a narrow area or in short-distance pipelines. The limitation of the above methods can be partially overcome by the use of the pressure-change- based technique (Vitkovský, Lambert, Simpson, \& Liggett, 2007) (Ghazali, Staszewski, 


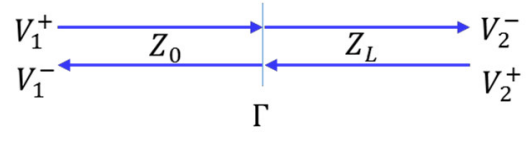

Figure 1. Impedance disparity and reflected coefficient

Shucksmith, Boxall, \& Beck, 2010) (Covas, Ramos, \& De Almeida, 2005) (Brunone, 1999). The method is applicable to detect leaks in a wide area and is appropriate to a pipeline treating high water pressure (Puust, Kapelan, Savic, \& Koppel, 2010).

Recently, a method based on time-domain reflectometry (TDR) (Kim, Suh, Cho, Singh, \& Seo, 2015) (Cataldo, Cannazza, De Benedetto, \& Giaquinto, 2012) was proposed to reliably detect leaks from water pipelines. The TDR-based leak detection method can inform observers about locations of leaks through the measurement of a reflected signal on a transmission line which is installed on or near the pipelines. The unique advantage of the method is to detect the existence of one or multiple leaks and to locate their sites (Kim, Woo, Youn, \& Huh, 2015). However, it should be noted that the time required for modeling the TDR signal becomes lengthy for the applications of long-distance pipelines. As the pipeline length increases, the amount of the computational time for training increases. For example, it is almost infeasible to detect leaks by human inspection for Los Angeles Aqueduct whose length is about 670 kilometers. Therefore, there is room for further improvement of the TDR-based leak detection method to applications in long- distance pipelines.

In this research, we attempted to develop a leak detection system that can be applicable to condition monitoring of long-distance pipelines. An S-parameter model is combined with the principle of TDR to relieve computational burden of the existing TDR models. It is expected that the proposed Sparameter based model significantly reduce the computational time to model the TDR signal in long distance pipelines and multiple leaks condition. The performance of the proposed model is evaluated through laboratory experiments.

The rest of the paper is organized as follows. The review of existing TDR-based leak detection methods is explained in Section 2. In Section 3, the S-parameter based model is proposed. In Section 4, case studies are described to validate the accuracy and efficiency of the proposed model. Moreover, incorporating with the proposed model, the TDR-based leak detection system is demonstrated. Finally, Section 5 gives conclusions.

\section{REVIEW OF TDR-BASED LEAK DETECTIONMETHODS}

Existing TDR-based leak detection methods is overviewed in this section. The principle of the TDR technique is first explained. Then, existing TDR-based leak detection methods are described according to the way it is used. Finally,

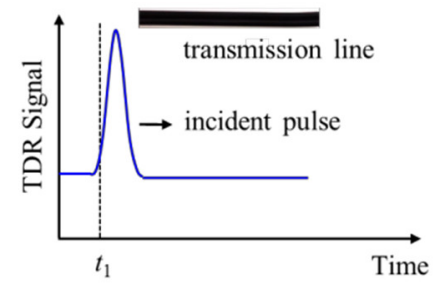

(a)

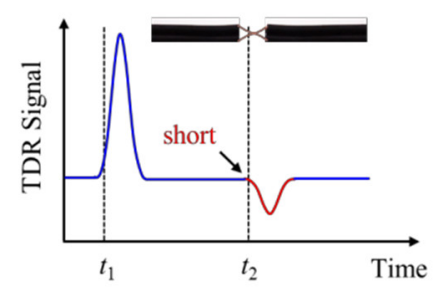

(b)

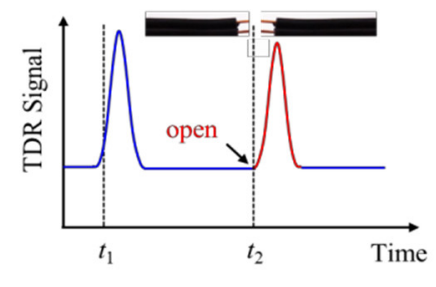

(c)

Figure 2. TDR signal for electrical: (a) normal, (b) open, and (c) short

limitations and challenges that prohibit the existing TDR technique from implementing in real applications are presented.

\subsection{Principles of TDR}

The TDR is defined as the technique that analyzes conditions of a transmission line through observing the reflected waveforms (O'Connor, \& Dowding, 1999). An incident pulse generated by a TDR instrument is propagated along the transmission line. The incident pulse is reflected when it meets a fault on the line. The reflected pulse is monitored by the TDR instrument. The mechanism of the reflection is the impedances of discontinuity in the transmission line as shown in Fig. 1. The impedance mismatch between $Z_{0}$ and $Z_{L}$ is referred to as the reflection coefficient:

$$
\Gamma=\frac{Z_{L}-Z_{0}}{Z_{L}+Z_{0}}=\left[\frac{V_{1}^{-}}{V_{1}^{+}}\right]_{V_{2}^{+}=0}
$$

where $Z$ is the characteristic impedance and $\Gamma$ is the reflection coefficient whose value is between negative and positive ones, not zero. If a $\Gamma$ is a negative value, the shape of the 


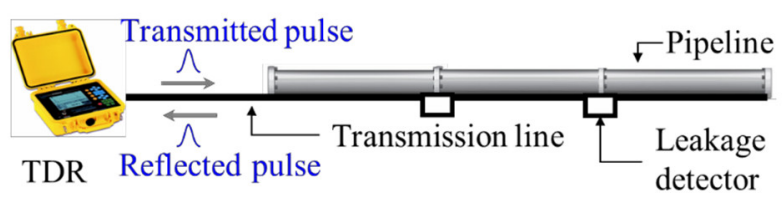

Figure 3. Concept of leak detection using TDR

reflected pulse is upside down for the incident pulse. If the value of $\Gamma$ is one, the shape of the reflected pulse is the same as that of the incident pulse, which indicates electric open, as shown in Fig. 2. The TDR technique can also locate faults on the transmission line by calculating the velocity of propagation and pulse traveling time, as in Eq. (2). The distance $(L f)$ between the fault and the TDR instrument is defined:

$$
L_{f}=v_{p} \frac{t_{2}-t_{1}}{2}
$$

where $t_{1}$ is the incident time when the pulse starts to transmit into the line and $t_{2}$ is the arrival time when the reflected pulse is measured at the TDR instrument. $v_{p}$ is the velocity of propagation of the traveling pulse on the transmission line. As using these principles, the TDR method can detect faults such as electrical open, short, or chafe in the transmission lines. The locations of their faults can be also found.

Moreover, many researchers utilize the TDR technique in various areas, such as for monitoring the health state of electronic devices (Yang, Choi, Lee, Ten, \& S. Lim, 2008) (Kwon, Azarian, \& Pecht, 2009), monitoring bridge scour (Yu, Zhang, Tao, \& Yu, 2013) (Yu, \& Yu, 2009), measuring moisture of soil (Calamita, Brocca, Perrone, Piscitelli, Lapenna, \& Melone, 2012) (Ledieu, De Ridder, De Clerck, $\&$ Dautrebande, 1986) and estimating the amount of fluid in a tank (Di Sante, 2005). In addition, the TDR technique is applied as a leak detection system recently.

\subsection{TDR-based Leak Detection Methods}

The TDR-based leak detection methods have been developed by correlating water leakage in the pipeline with the change of impedance in the transmission line. The impedance is determined by electric properties of transmission line such as Eq. (3).

$$
\mathrm{Z}=\sqrt{\frac{R+j \omega L}{G+j \omega C}}
$$

where $R$ is resistance, $L$ is inductance, $C$ is capacitance, and $G$ is conductance of the transmission line. These $R, L, C$, and $G$ are dependent on electric characteristics of dielectric materials including permittivity, conductivity, and permeability. The dielectric material exists between conduction wires of a transmission line or around the wires (Reinhold, \& Pavel, 2000). If the dielectric material is wet by leaked water, its electric characteristics are shifted.

Several TDR-based leak detection methods were developed in the past (Kim et al. ,2015) (Cataldo et al., 2012) (Kim et al., 2015). A dielectric-change-based method uses electrical characteristics of dielectric materials such as soil around the transmission line. In this method, the transmission line is buried near the pipelines. Leakage water from the pipe soaks the dielectric materials and changes the electrical characteristics of its. The impedance of the transmission line with the wet material is changed in the leakage section. This change of impedance causes change of reflection coefficient in the transmission line. Through observation of the change of reflection coefficient in the measured TDR signal, a location of the leak is inferred (Cataldo et al., 2012). However, this method has a limitation that electrical characteristics can be changed not only the leakage water but also by other water, such as rainwater, underground water, and moisture in the ground.

There is also a leakage-detector-based method. The method is based on the detection of an electric short at leakage sector in the transmission line. To generate the electric short, the special device, so-called 'leakage detector', is used in this method. The device can be connected to the transmission line and causes electric short when the leakage water soaks into itself. The electric short also causes change of impedance of the transmission line. The transmission line with the devices is attached on the pipeline as like Fig. 3. This method is able to acquiring much stronger reflected signal than it of other methods without the device (Kim et al., 2015). But this method has a signal analyzing issue. In case of multiple leaks, pulses reflected from each of leaks are overlapped in the TDR signal. The measured TDR signal is limited to be interpreted by a visual inspection or a threshold criteria based inspection.

The other is a model-based method. In terms of a signal acquisition way, this method is same as the method using the leakage detector. However, this model-based method is different in terms of interpreting the signal. Unlike a visual inspection or a threshold criteria inspection of other methods, this method uses a forward model and an inverse model to find locations of leaks. The purpose of the forward model models a TDR signal according to inputted leak information, such as locations. The forward model calculates voltages on the transmission line per unit time of the TDR equipment and is induced by using an RLCG circuit model in the lossy transmission line (LTL) theory on the finite-difference time domain (FDTD). On the contrary to this, the inverse model infers the leak information using a measured TDR signal. The inverse model also employed the Bayesian inference to inter leak information. Applying Bayesian inference improves detecting ability in light of the uncertainties of the model and errors of measurement (Wang, Youn, Xi, \& Kloess, 2009), (Wang, Youn, \& Hu, 2012). It was shown that the method is 


\begin{tabular}{|c|c|c|}
\hline Methods & Pros & Cons \\
\hline $\begin{array}{l}\text { Dielectric- } \\
\text { change- } \\
\text { based } \\
\text { method }\end{array}$ & $\begin{array}{l}\text { It is easy to } \\
\text { install into existing } \\
\text { buried pipelines. }\end{array}$ & $\begin{array}{l}\text { Reflection } \\
\text { waveforms is occurred } \\
\text { for not only water } \\
\text { leakage but also outside } \\
\text { water. } \\
\text { It is difficult to } \\
\text { detect multiple leaks. }\end{array}$ \\
\hline $\begin{array}{l}\text { Leakage- } \\
\text { detector- } \\
\text { based } \\
\text { method }\end{array}$ & \begin{tabular}{l}
\multicolumn{1}{c}{ Reflection } \\
waveforms is \\
occurred for only \\
water leakage.
\end{tabular} & $\begin{array}{l}\text { It is difficult to } \\
\text { detect multiple leaks. } \\
\text { It is difficult to be } \\
\text { applied to existing } \\
\text { buried pipelines. }\end{array}$ \\
\hline $\begin{array}{l}\text { Model- } \\
\text { based } \\
\text { method }\end{array}$ & $\begin{array}{l}\text { Reflection } \\
\text { waveforms is } \\
\text { occurred for only } \\
\text { water leakage. } \\
\text { It is able to } \\
\text { detect } \\
\text { multiple leaks. }\end{array}$ & $\begin{array}{l}\text { Detection range can } \\
\text { be limited a length of } \\
\text { pipeline by a modeling } \\
\text { method. } \\
\text { It is difficult to be } \\
\text { applied to existing } \\
\text { buried pipelines. }\end{array}$ \\
\hline
\end{tabular}

Table 1. Pros and Cons of TDR-based Methods

robust against peripheral noise and superior in signal interpretability for multiple leaks (Kim et al., 2015).

\subsection{Limitations of the Existing Methods for Long- Distance Pipe Leakage Detection}

Pros and cons of some existing methods are summarized in Table 1. The existing model-based method has limitations to apply to long distance water distribution pipelines. The problem is related to computational efficiency of the forward model. If the computational efficiency of the forward model is low, the process of building the sample data set is a timeconsuming task. Especially, the longer the pipeline is, the more the size of the sample data set increases. In some case, the sample data set may not be able to be built in theallotted construction period. This limitation can be understood through a following example.

Suppose a five kilometer pipeline is assumed to be connected of 10 meter pipes and be 499 leakage detectors are installed on the connecting parts. The number ofsamples for possible leak situations can be calculated as:

$$
N_{\text {sample data }}=\left(\sum_{i=1}^{K} C\left(N_{\text {detector }}, i\right)\right)+1
$$

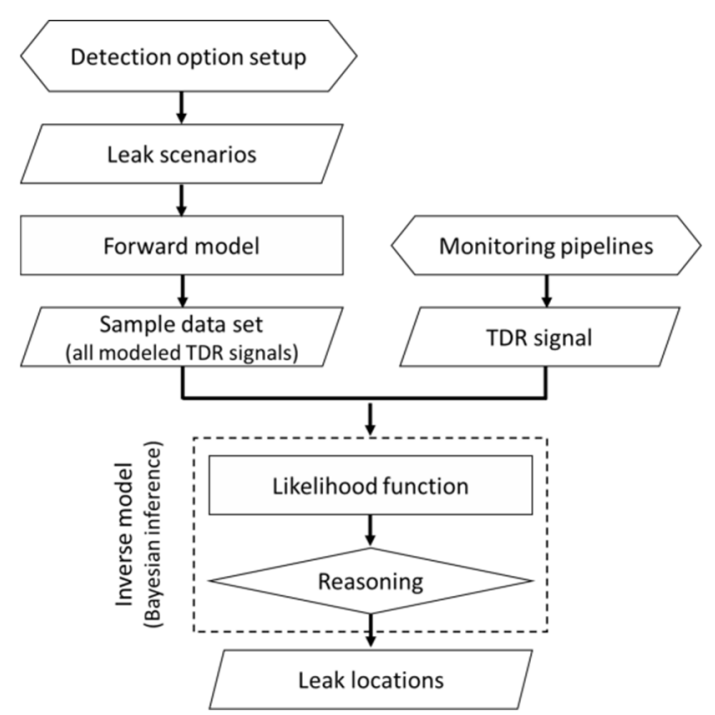

Figure 4. Framework for the model-based leak detection method

$$
T_{\text {tot }}=N_{\text {sample data }} \cdot t_{\text {sample }}
$$

where $N_{\text {sample data }}$ is the number of samples of sample data set, $K$ is the number of maximum multiple detectability at the same time, $N_{\text {detector }}$ is the number of detectors, $T_{\text {tot }}$ is the total time to build sample data set, tsample is the time to build one sample, and $C$ is combination operator. Through the combination operator, the number of all possible leak situations is calculated. The last term of " +1 " adds to the formula to account for the case of a normal situation without any leaks.

If the number of maximum detectability is set as three leaks, the sample data set is consisted of 20,709,000 samples by Eq. (4). A time to build one sample signal of the RLCG based forward model is 3.8138 seconds, which was performed by personnel computer with Intel Core i5 3.1 GHz processor and eight GRAM. The total time to build is two and a half years by Eq. (5). This result indicates that the model-based method is not applicable to leak detection of long distance water pipelines. Thus, the computationally efficient forward model must be developed to practically apply the model based leak detection method to the real water distribution system.

\section{S-PARAMETER-BASED FORWARD MODEL FOR LEAK DETECTION}

As shown in Fig. 4, the framework for leak detection consists of (1) construction of a sample data set, (2) comparison the sample data set with a measured TDR signal, and (3) reasoning on locations of leaks (Kim et al., 2015). The first step is to build sample data sets (i.e., emulated TDR signals) using a forward model that accounts for all possible leak situations. The second step is to compare a measured TDR signal with each of the emulated TDR signals from the 
sample data set. A likelihood metric can be used for quantifying the degree of similarity. The last step is to find locations of leaks by Bayesian inference and the likelihood metric.

This study focuses on the first step to address the problem about computational efficiency of the existing RLCG based forward model. To resolve the computational challenge, the frequency domain-based TDR signal modeling method, namely S-parameter-based forward modeling, is newly adopted for pipeline leak detection. TDR signals are modeled in the time domain in the RLCG-based forward modeling, whereas they can be also modeled in the frequency domain. The frequency domain-based modeling transforms a timeseries TDR signal into a frequency-domain signal. In the frequency domain, calculation required to find the location of leaks are conducted. Then, the frequency-domain signal is transformed back into a time-domain signal by the inverse Fourier transform. In Section 3.1, the physical concept of Sparameter is described. In Section 3.2, the process of inducing the S-parameter based forward model is explained.

\subsection{S-parameter}

The S-parameter is a frequency-domain measure that quantifies the transmissibility of the power of electrical signals between an input and an output of a physical segment (Reinhold, \& Pavel, 2000). The S-parameter is formulated (Reinhold et al., 2000):

$$
S_{i j}=\left.\frac{V_{i}^{-}}{V_{j}^{+}}\right|_{V_{i}^{+}=0}=\frac{\text { reflected voltage }}{\text { incident voltage }}
$$

The S-parameters of a two-port segment in the transmission line are depicted in Fig. 5. The segment is a part of a transmission line that is adhered on a pipeline. The port means a gate through which the electrical signal enters and leaves. The inlet and outlet ports are defined in accordance with the direction of water flow in the pipeline. An input electrical signal is divided into a transmitted signal and a reflected signal by electric characteristics of the segment. A ratio of reflection to transmission of the electric signal is determined by S-parameters of the segment. The relationship between voltages and S-parameters is defined:

$$
\left[\begin{array}{c}
V_{\text {in }}^{-} \\
V_{\text {out }}^{-}
\end{array}\right]=\left[\begin{array}{cc}
S_{\text {in }, \text { in }} & S_{\text {in,out }} \\
S_{\text {out }, \text { in }} & S_{\text {out }, \text { out }}
\end{array}\right]\left[\begin{array}{c}
V_{\text {in }}^{+} \\
V_{\text {out }}^{+}
\end{array}\right]
$$

Eq. (7) explains how $V_{\text {in }}^{-}$and $V_{\text {out }}^{-}$is composed of. $V_{\text {in }}^{-}$is the sum of two pulses; one is reflected pulse of $V_{i n}^{+}$at inlet port and the other is $V_{\text {out }}^{+}$passing through the outlet port as in Fig. 5. A generalized relation between the $\Gamma$ and S-parameters is defined (Reinhold et al., 2000):

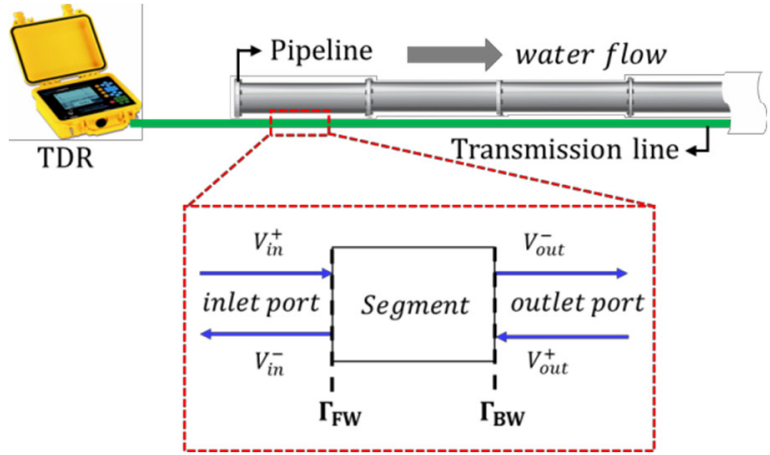

Figure 5. Concept of S-parameters in the segment

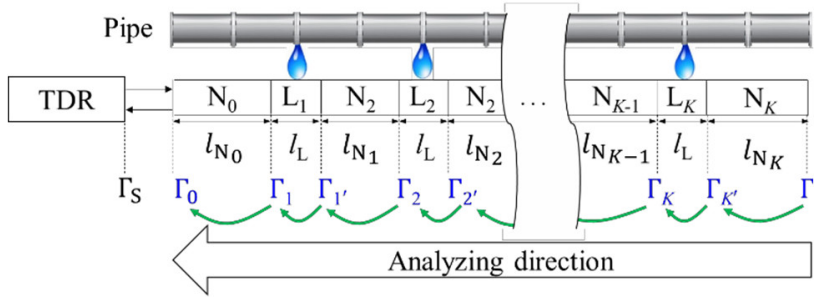

Figure 6. Schematic of a leak detection system with normal and leakage segments (Ni: normal segment; Li: leakage segment)

$$
\Gamma_{\mathrm{FW}}=S_{\text {in, in }}+\frac{S_{\text {in,out }} S_{\text {out }, \text { in }} \Gamma_{\mathrm{BW}}}{1-S_{\text {out }, \text { out }} \Gamma_{\mathrm{BW}}}
$$

where $\Gamma_{\mathrm{FW}}$ and $\Gamma_{\mathrm{BW}}$ is the forward and backward reflection coefficient at inlet port and outlet port, respectively. That is, $\Gamma_{\mathrm{FW}}$ is the ratio of $V_{i n}^{-}$to $V_{i n}^{+}$. Similarly, $\Gamma_{\mathrm{BW}}$ is explained as the ratio between $V_{\text {out }}^{-}$to $V_{\text {out }}^{+}$. Eq. (8) is merely the reformulation of that using S-parameters and $\Gamma_{\mathrm{BW}}$.

\subsection{Derivation of S-parameter-based Forward Model}

This section presents the development of the S-parameterbased forward model to emulate the frequency-domain TDR signals from normal leakage segments. As shown in Fig. 6, the transmission lines attached on pipeline bonds is grouped into normal and leakage segments. A leakage segment, $\mathrm{L} i$, is defined as the part where a leak is detected by the leakage detector installed on the pipeline bond with leakage water. The normal segment, $\mathrm{N} i$, is defined as the part where no leak is detected. The locations of normal and leakage segments are dependent on various leak scenarios. Consequently, reflection coefficients at the boundary of the segments vary. In Sections 3.2.1 and 3.2.2, the S-parameters of normal and leakage segments are described, respectively. In Section 3.2.3, the S-parameter based forward model with normal and leakage segments is constructed. 


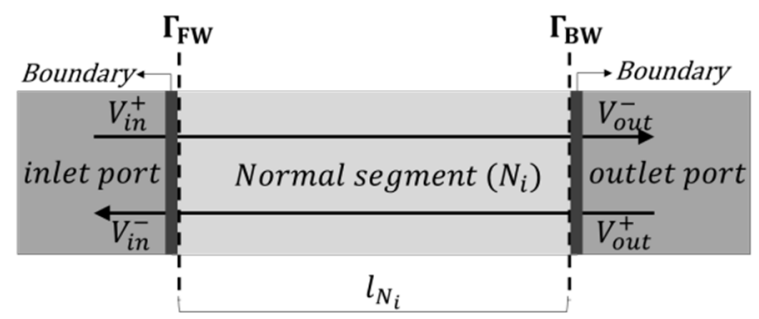

(a)

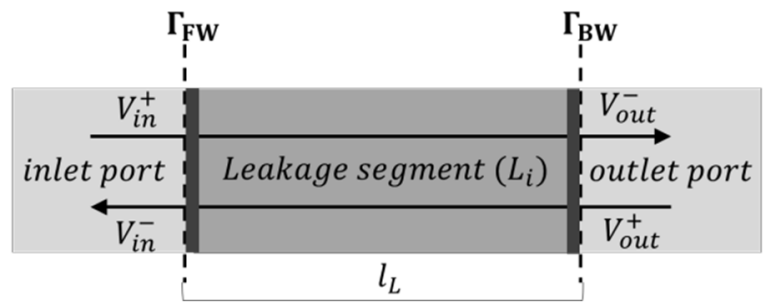

(b)

Figure 7. Reflection coefficients with S-parameters for: (a) Normal segment and (b) leakage segment

\subsubsection{Normal segment}

As shown in Fig. 7(a), the normal segment is defined as the part that excludes the boundaries at both sides. As there is no change of impedance in the normal segment, no electric reflection happens in the normal segment. This indicates that input electrical signals, $V_{\text {in }}{ }^{+}$or $V_{\text {out }}{ }^{+}$, are not reflected back into the same ports. Therefore, the S-parameters related to reflection, $S_{i n, \text { in }}^{N_{i}}$ and $S_{\text {out,out }}^{N_{i}}$, are zero in the normal segment:

$$
\mathrm{S}_{\text {in }, \text { in }}^{N_{i}}=S_{\text {out }, \text { out }}^{N_{i}}=0
$$

However, although there is no reflection in the normal segment, there is the electric signal attenuation along the transmission line. The amount of the loss is in proportion to the length of the segment. The S-parameters related to transmissibility, $\mathrm{S}_{\text {in,out }}^{N_{i}}$ and $S_{\text {out,in }}^{N_{i}}$, are not zero but formulated as:

$$
\mathrm{S}_{\text {in }, \text { out }}^{N_{i}}=S_{\text {out }, \text { in }}^{N_{i}}=e^{-\gamma l_{N_{i}}}
$$

where $\gamma$ is the propagation constant of the segment and $l_{N i}$ is the length of the segment. As described earlier, the magnitude of the transmitted signal attenuates and its phase changes. $\gamma$ represents the propagation characteristic of the signal in a transmission line that is determined by electrical properties including $\mathrm{R}$, L, C, and G, of a transmission line:

$$
\gamma=\sqrt{[R+j \omega L][G+j \omega C]}
$$

where $\omega$ is the sampling frequency in the TDR measurement; R, L, G, and C are electrical properties of a cable (See Table 2 for an example of two parallel cables.).

The electrical transmissibility of the normal segment can be derived by putting Eqs. (9) and (10) into Eq. (8). $\Gamma_{\mathrm{FW}}$ for the normal segment becomes a function of the length of the segment and $\Gamma_{\mathrm{BW}}$.

$$
\Gamma_{\mathrm{FW}}=e^{-2 \gamma l_{N_{i}} \cdot \Gamma_{\mathrm{BW}}}
$$

\subsubsection{Leakage segment}

As shown in Fig. 7(b), the leakage segment is defined as the part that includes the boundaries at both sides. Electric reflection happens in the leakage segment because the impedance changes across the segment. Different from the reflection characteristic of the normal segment, some portion of the input electrical signals, $V_{\text {in }}{ }^{+}$or $V_{\text {out }}{ }^{+}$, are reflected back into the same ports. Consequently, S-parameters related to the reflection, $S_{i n, i n}^{L_{i}}$ and $S_{\text {out,out }}^{L_{i}}$, are not zero. As an example, $S$ parameter for a leakage segment in a general transmission line is given by Stefan Schuet et al (Schuet, Timucin, \& Wheeler, 2011):

$$
\mathrm{S}_{\text {in }, \text { in }}^{L_{i}}=\mathrm{S}_{\text {out }, \text { out }}^{L_{i}}=\frac{\Gamma_{\mathrm{BW}}\left(e^{-j \omega 2 t_{d}}-1\right)}{1-\Gamma_{\mathrm{BW}}^{2} e^{-j \omega 2 t_{d}}}, \quad\left(t_{d}=l_{L} / v_{p}\right)
$$

where $t_{d}$ is travel time of pulse when the pulse passes the leak segment; $l_{L}$ is the length of the leakage segment (i.e., length of the leakage detector in every leakage segment); and $v_{p}$ is the propagation velocity of the pulse.

For the calculation of $S_{\text {in,out }}^{L_{i}}$ and $\mathrm{S}_{\text {out }, \text { in }}^{L_{i}}$, the reflection at the both boundaries and electric loss should be considered. These S-parameters are defined as in Eq. (16) which is also established by Stefan Schuet et al (Schuet et al, 2011):

$$
\mathrm{S}_{\text {in }, \text { out }}^{L_{i}}=\mathrm{S}_{\text {out }, \text { in }}^{L_{i}}=\frac{\left(1-\Gamma_{\mathrm{BW}}^{2}\right) e^{-j \omega 2 t_{d}}}{1-\Gamma_{\mathrm{BW}}^{2} e^{-j \omega 2 t_{d}}}
$$

Thus, the electrical transmissibility of the leakage segment can be derived by putting Eqs. (13) and (14) into Eq. (8). Moreover, this $\Gamma_{\mathrm{FW}}$ for the leakage segment is also determined by the length of the segment and $\Gamma_{\mathrm{BW}}$ like the normal segment. 


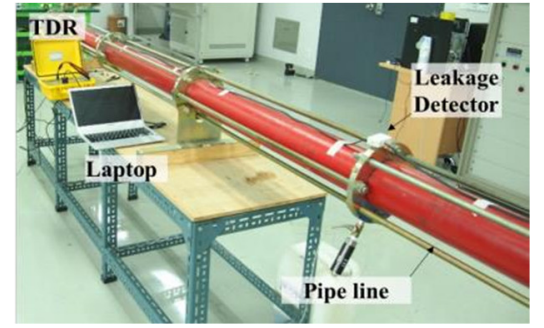

Figure 8. Experimental set up for validating the forward model

$$
\Gamma_{\mathrm{FW}}=\frac{\Gamma_{\mathrm{BW}}\left(e^{-j \omega 2 t} d-1\right)}{1-\Gamma_{\mathrm{BW}}^{2} e^{-j \omega 2 t} d}+\frac{\left\{\frac{\left(1-\Gamma_{\mathrm{BW}}^{2}\right) e^{-j \omega 2 t} d}{1-\Gamma_{\mathrm{BW}}^{2} e^{-j \omega 2 t} d}\right\}^{2} \cdot \Gamma_{\mathrm{BW}}}{1-\left\{\frac{\Gamma_{\mathrm{BW}}\left(e^{-j \omega 2 t} d-1\right)}{1-\Gamma_{\mathrm{BW}}^{2} e^{-j \omega 2 t} d}\right\} \cdot \Gamma_{\mathrm{BW}}}
$$

\subsubsection{Segment integration}

To build the S-parameter-based forward model, the reflection coefficients that represent each of segments should be integrated. As show in Fig. 6, segments are continuously connected and $\Gamma_{\mathrm{BW}}$ of each segment overlaps with $\Gamma_{\mathrm{FW}}$ of its backward segment. It means that $\Gamma_{\mathrm{FW}}$ of each segment is to be $\Gamma_{\mathrm{BW}}$ of its front segment. For example, the $\Gamma_{\mathrm{FW}}$ of $\mathrm{N}_{K}$ is same with the $\Gamma_{\mathrm{BW}}$ of $\mathrm{N}_{K-1}$. Thus, $\Gamma_{0}$, the reflection coefficient at zero meter of the transmission line, is calculated as Eqs. (16) to (19). This process of the calculation only repeats Eq. (12) and Eq. (15). Moreover, the value of $\Gamma_{\mathrm{E}}$ equals to one as it is the reflection coefficient of the open circuit at the end point of the transmission line.

$$
\begin{gathered}
\Gamma_{K^{\prime}}=e^{-2 \gamma l_{N_{k}} \cdot \Gamma_{\mathrm{E}}} \\
\Gamma_{K}=S_{\text {in }, \text { in }}^{L_{K}}+\frac{S_{\text {in,out }}^{L_{K}} S_{\text {out }, \text { in }}^{L_{K}} \Gamma_{K^{\prime}}}{1-S_{\text {out }, \text { out }}^{L_{K}} \Gamma_{K^{\prime}}} \\
\Gamma_{1}=S_{\text {in }, \text { in }}^{L_{1}}+\frac{S_{\text {in,out }}^{L_{1}} S_{\text {out }, \text { in }}^{L_{1}} \Gamma_{1^{\prime}}}{1-S_{\text {out }, \text { out }}^{L_{1}} \Gamma_{1^{\prime}}} \\
\Gamma_{0}=e^{-2 \gamma l_{N_{0}} \cdot \Gamma_{1}}
\end{gathered}
$$

The lengths of each segment is reflected in the calculated $\Gamma_{0}$ because S-parameters of each steps include lengths of segments which are dependent on leak locations. Thus, the $\Gamma_{0}$ includes the information about leak locations. Using the $\Gamma 0$, a transfer function, $H$, can be defined (Schuet et al, 2011):

$$
H(\boldsymbol{L}, \boldsymbol{\omega})=\frac{V_{M}(\boldsymbol{L}, \boldsymbol{\omega})}{V_{S}(\boldsymbol{\omega})}=\frac{G}{2}\left(1+\frac{\Gamma_{\mathrm{S}}+\Gamma_{0}(\boldsymbol{L})}{1+\Gamma_{\mathrm{S}} \Gamma_{0}(\boldsymbol{L})} e^{-j 2 \boldsymbol{\omega} t_{M}}\right)
$$

where $\boldsymbol{L}$ is the random variable that indicates the location of leaks; $V_{M}(\boldsymbol{L}, \boldsymbol{\omega})$ is the measured voltage from a TDR instrument in the frequency domain; $V_{S}(\boldsymbol{\omega})$ is the source voltage generated by the TDR instrument; $G$ is the gain factor; $\Gamma_{\mathrm{S}}$ is the reflection coefficient between the TDR instrument and the transmission line; and $t_{M}$ is the internal time delay in the TDR instrument.

All the parameters except $\Gamma_{0}(\boldsymbol{L})$ are constants that can be determined from specifications of the TDR equipment and electrical properties of a transmission line. Thus, $H(\boldsymbol{L}, \boldsymbol{\omega})$ is only dependent on $\Gamma_{0}(\boldsymbol{\theta}) . V_{M}(\boldsymbol{L}, \boldsymbol{\omega})$ is defined the operation of the transfer function and the fast Fourier transform (FFT) of the source voltage in the time domain, $v_{S}(t)$ :

$$
V_{M}(\boldsymbol{L}, \boldsymbol{\omega})=H(\boldsymbol{L}, \boldsymbol{\omega}) \odot F F T\left(v_{S}(\boldsymbol{t})\right)
$$

where $\odot$ is the element-by-element vector multiplication operation and $\boldsymbol{t}$ is the measurement time. The time domain signal $\left(v_{M}(\boldsymbol{L}, \boldsymbol{t})\right)$ is obtained by inverse fast Fourier transform (IFFT) of $V_{M}(\boldsymbol{L}, \boldsymbol{\omega})$ :

$$
v_{M}(\boldsymbol{L}, \boldsymbol{t})=\operatorname{IFFT}\left(V_{M}(\boldsymbol{L}, \boldsymbol{\omega})\right)
$$

$v_{M}(\boldsymbol{L}, \boldsymbol{t})$ emulates the actual TDR signal tha is reflected back from the transmission line with water leaks, which is the outcome from the S-parameter based forward model for leak detection.

\section{Case Study}

The performance of the proposed S-parameter-based forward model is demonstrated with a case study. In Section 4.1, the experimental setup is described. The experimental set up is then demonstrated by applying a real leak situation to the test bed. In Section 4.2, parameters of the proposed model in Section 3 are estimated. To estimate the parameters, reported literature values and experimental measurement values are applied. Moreover, to reduce uncertainties, an optimization algorithm is used. In Section 4.3, the validity of the model is evaluated in terms of accuracy and computational cost for building sample data set. Especially, these validations are also evaluated by comparing to the existing RLCG-based forward model. In section 4.4, the advantage of the proposed forward model is discussed.

\subsection{Experimental Setup}

A custom test bed was designed at the lab scale, as shown in Fig. 8. This system is comprised of three parts, including the pipeline, the leak detection system, and a data acquisition system. First, the pipeline is made up of three meters long pipes with 12 centimeter radius and an outer housing case at 


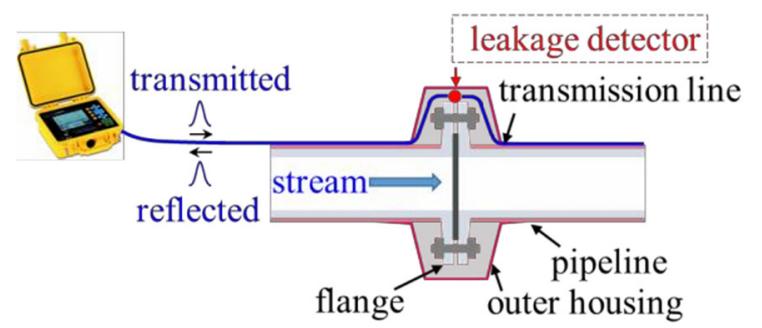

(a)

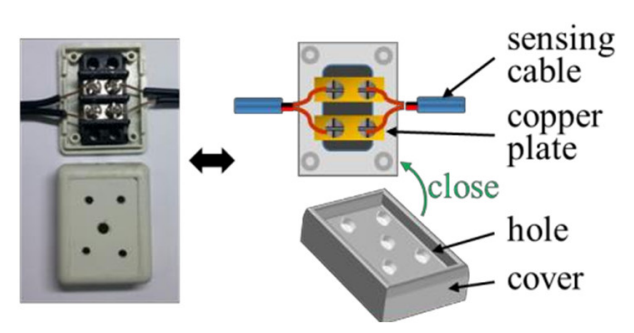

(b)

Figure 9. Leak detection system: (a) system components, (b) leakage detector

the joints. The outer housing case is installed around joints where leakage is likely to occur. The housing plays the role of a reservoir for the leakage water to ensure the leak detector gets wet. This wetness causes an electrical short and the reflection occurs in the soaked detector. Second, the leak detection system is comprised of transmission line and leak detector. The transmission line is a twin parallel cable that is made from copper wire with 0.4 millimeter radius is used as the transmission line in this experimental setup. Recently, pipes with embedded the transmission line have been produced, so they can be installed with the proposed method in the real water distribution system. The leak detector consists of two copper plates and a plastic case with holes, as shown in Fig. 9. The detector is isolated from external moisture by the outer housing, and is thus only affected by leaking water at the joint. The copper plate is also exposed to contact with the leaking water; the water then plays the role of a conductor between the two plates. Third, the data acquisition part is composed of the TDR instrument and a laptop. The model of TDR instrument in the test setup is an mTDR-070 from Nanotronics Corp. with an input bandwidth of $300 \mathrm{MHz}$, output pulse of two volts, rising time of $1 \mathrm{~ns}$, maximum effective distance of 20 kilometers, and a characteristic impedance of $75 \Omega$. The laptop specifications include an Intel core i5 3.1 GHz processor with eight GRAMs. The TDR instrument is connected to the start of the sensing cable to transmit the pulse and receive the reflected pulse. The laptop is connected also to the TDR instrument for analyzing the acquired data. The experimental setup only focuses on the leakage at connection area. Although leaks can occur on the any points of the pipeline, this research considers

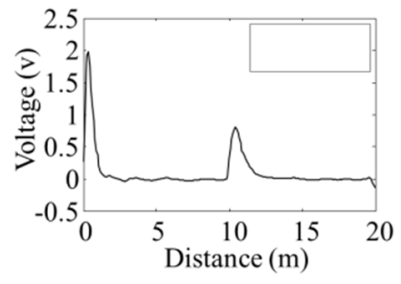

(a)

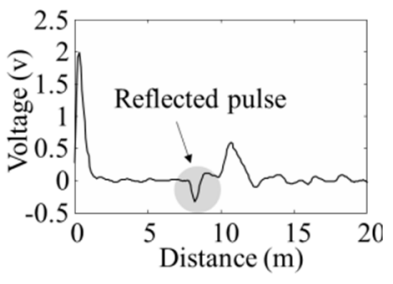

(b)
Figure 10. Operation check of the leak detector: (a) no leak, (b) single leak at $8 \mathrm{~m}$

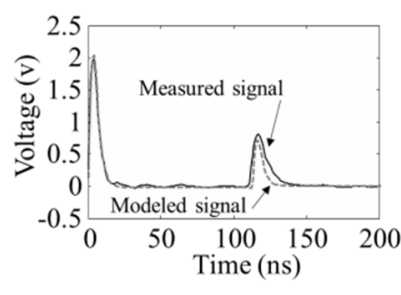

(a)

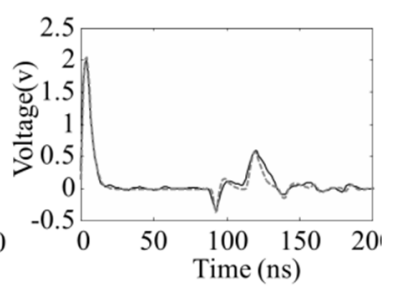

(b)
Figure 11. Comparison between measured signal and modeled signal: (a) no leak, (b) single leak at $8 \mathrm{~m}$

on the leakage at pipeline connections. The suggested detection method can be applied to any region of pipelines if the leak detection system is installed on the pipeline body.

Operation performance of this system was demonstrated through a simple experiment that was conducted using a 10 meters cable with one leak detector installed eight meters along the cable. Then, the leak detector was attached to the flange and a leakage situation was applied to the system. In the normal situation (no leakage), the reflection pulse about an electrical open is observed at 10 meters as shown Fig. 10 (a). In the single leak situation, however, the reflection pulse is observed at the leak location, eight meters, as shown in Fig. 10 (b). And then, to estimate model parameters and evaluate accuracy and computational cost of the developed model, the experiment results are needed under multiple leak scenarios such as single, double, and triple leaks. To conduct the experiment for multiple leaks scenarios, tests were manually performed to intentionally change the locations of leaks.

\subsection{Model Parameters Estimation}

Before validating the developed forward model in Section 3, unknown parameters of the model should be estimated to increase accuracy of the model. To precisely estimate, sample signals which are acquired from the built test-bed and an optimization function are used. The sample signals are measured under no leak and single leak situations as shown Fig. 11. Targets of estimation are a velocity factor of the used transmission line, electrical properties of dielectric of the used 


\begin{tabular}{c|c|c}
\hline Parameter & Initial value & Estimated value \\
\hline $\mathrm{V}_{\mathrm{F}}$ & 0.597 & 0.597 \\
\hline$\mu_{d}[\mathrm{H} / \mathrm{m}]$ & $1.25 \mathrm{e}-6$ & $1.5 \mathrm{e}-6$ \\
\hline$\varepsilon_{d}[\mathrm{~F} / \mathrm{m}]$ & $1.86-12$ & $2.12 \mathrm{e}-12$ \\
\hline$\sigma_{d}[\mathrm{~S} / \mathrm{m}]$ & $1 \mathrm{e}-23$ & $1.15 \mathrm{e}-4$ \\
\hline$t_{M}[\mathrm{~s}]$ & $1 \mathrm{e}-10$ & $1 \mathrm{e}-10$ \\
\hline
\end{tabular}

Table 3. Model parameters estimation

transmission line, and unknown specifications of the TDR equipment. The velocity factor, $\mathrm{V}_{\mathrm{F}}$, is the ratio of the speed in comparison with the speed of light in a vacuum. Thus, the $\mathrm{V}_{\mathrm{F}}$ is an important parameter to acquire the $v_{p}$ of the applied transmission line. The electrical properties of the used transmission line, such as $\mu_{d}, \varepsilon_{d}$, and $\sigma_{d}$, are also important parameters to calculate the $\mathrm{R}, \mathrm{L}, \mathrm{G}$, and $\mathrm{C}$ of the used transmission line. Unlike a coaxial cable, dielectric material of the two-parallel cable is not constant because it is combined with insulators like rubber and air. The unknown specifications of the TDR equipment are $t_{M}$ which is also important parameters to increase accuracy of the developed model. Initial values of these unknown parameter are set through measurements and research reviews. The $V_{F}$ is referred to a general measurement method through a simple experiment using a reference transmission line like Eq. (23).

$$
V_{F}=\frac{2 \cdot L_{R e f} \cdot \Delta t}{c}
$$

where $\mathrm{L}_{\mathrm{Ref}}$ is a length of the reference transmission line; $\Delta \mathrm{t}$ is a travel time of pulse when the pulse has round-trip in the reference transmission line; $\mathrm{c}$ is the velocity of the light.

The dielectric properties are referred to properties of the Teflon because it is generally suitable for use as an insulator in cables. The $t_{M}$ of the equipment is referred to similar research paper (Schuet et al, 2011). Table 3 shows the initial values of these unknown parameters. In order to decrease each error of the initial values of the parameters, parameter optimization process is conducted by using the least square method (LSM) (Miller, 2006). Table 3 shows the estimated values of the parameters after conducting the optimization.

\subsection{Performance Evaluation of the Proposed Model}

The performance of the proposed forward model is evaluated in terms of (1) an accuracy aspect and (2) a computational cost aspect. To evaluate these aspects, the double leaks scenario is applied. The pipeline in the experimental setup is 10 meters long with two leaks at six meters and eight meters. As shown in Fig. 12, the S- parameter-based forward model accurately represents the actual TDR signal measured from the experiment. The modeled signal is also converted to the distance domain to arrive at more practical information. To

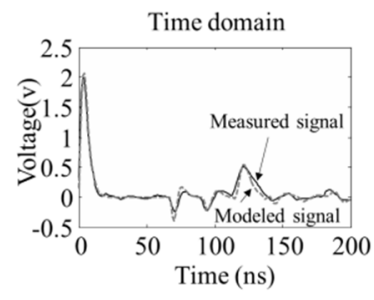

(a)

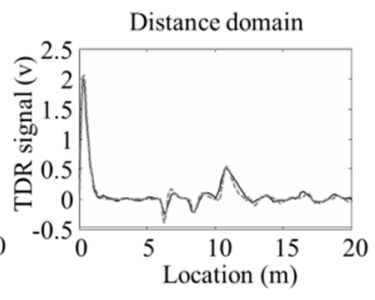

(b)
Figure 12. Validation of the forward model with two leaks $(6 \mathrm{~m}$ and $8 \mathrm{~m})$ on the $10 \mathrm{~m}$ cable: (a) time domain, (b) distance domain

\begin{tabular}{c|c|c}
\hline Measure [min - max] & $\begin{array}{c}\text { S-parameter } \\
\text { model }\end{array}$ & $\begin{array}{c}\text { RLCG } \\
\text { model }\end{array}$ \\
\hline $\begin{array}{c}\text { Correlation Coefficient } \\
{[-1-1]}\end{array}$ & 0.9873 & 0.9869 \\
\hline WIFac [0 - 1] & 0.8594 & 0.8518 \\
\hline
\end{tabular}

Table 4. Comparison of accuracy between Sparameter model and RLCG model

assure objectivity, the accuracy of the proposed model is also validated by qualitative evaluation with measures in the Table 4. The applied stochastic measures are Correlation Coefficient and Weighted Integrated Factor (WIFac) (Twisk, Spit, Beebe, \& Depinet, 2007). WIFac is a measure to judge degree of match between two data groups. The measure utilizes three characters (max value, location of peak value, and graph shape of data group) of each data group. The WIFac normalizes the result of values to the range between zero to one. As shown Table 4, the WIFac value falls a little short of one because of difference of magnitude of the signals caused by periphery noise and physical uncertainties included in this system. The Correlation Coefficient related to tendency of peaks of the signals is close to one. The peak locations of the signals are important factor for inferring the leak locations in this detection system. Moreover, the accuracy of the proposed forward model does not decrease in comparison with existing RLCG based forward model. Thus, the accuracy of the proposed forward model is validated through the double leak scenario.

To evaluate the computational cost, the double leaks scenario is also applied. The $t_{\text {sample }}$ of model is a specific indicator which well represents the computational efficiency under the given scenario. The $t_{\text {sample }}$ is calculated using the results of multiple leaks scenario. The lab setting limits the opportunity to variously extend the length of the installed pipeline. Thus, to examine extended pipeline, we assumed that the length of each pipe unit ranged from one meter to 0.1 meter under the given length of the installed pipeline, 10 meters. This assumption enabled to examine different numbers of flanges 


\begin{tabular}{|c|c|c|c|c|c|}
\hline \multicolumn{2}{|c|}{ Length of unit pipe } & $1 \mathrm{~m}$ & $0.5 \mathrm{~m}$ & $0.2 \mathrm{~m}$ & $0.1 \mathrm{~m}$ \\
\hline \multicolumn{2}{|c|}{$N_{\text {detector }}$} & 9 & 19 & 49 & 99 \\
\hline \multicolumn{2}{|c|}{$N_{\text {sample data }}$} & 130 & 1,160 & 19,650 & 161,800 \\
\hline \multirow{2}{*}{$\begin{array}{c}\text { S-parameter } \\
\text { model }\end{array}$} & $T_{\text {tot }}[\mathrm{s}]$ & 0.34 & 3.03 & 51.42 & 433.15 \\
\hline & $\begin{array}{c}t_{\text {sample }}[\mathrm{s}] \\
\left(T_{\text {tot }} \div N_{\text {sample data }}\right)\end{array}$ & 0.002615 & 0.002612 & 0.002617 & 0.002677 \\
\hline \multirow{2}{*}{ RLCG model } & $T_{t o t}[\mathrm{~s}]$ & 487.69 & $4,442.52$ & $74,839.69$ & $625,400.32$ \\
\hline & $t_{\text {sample }}[\mathrm{s}]$ & 3.751432 & 3.829759 & 3.808636 & 3.865268 \\
\hline
\end{tabular}

Table 5. The Ttot and tsample with three maximum detectable leaks, as predicted by each forward model

and allowed $N_{\text {detector }}$ to increase, achieving the same effect as extending the length of the pipeline. As shown in Table 5, $N_{\text {detector }}, N_{\text {training data }}, T_{\text {tot }}$ and $t_{\text {sample }}$ were acquired according to the length of each unit pipeline with three leaks as the number of maximum detectability. As a result, the average $t_{\text {sample }}$ of the S- parameter based model was approximately 0.00263 second, which is 1,449 times better than average $t_{\text {sample }}$ of RLCG based forward model. Moreover, Table 6 shows the estimated $T_{\text {tot }}$ for various lengths of pipeline as using Eq. (5). In case of RLCG based model, $T_{t o t}$ of 20 kilometer pipeline is 161 years which is an impractical time to complete building the sample data set in the allotted construction period. $T_{\text {tot }}$ of five kilometer pipeline is two years which means that the detection system is also difficult to complete building the sample data set in the given construction period. On the other hand, in case of S-parameter based model, $T_{t o t}$ of 20 kilometer

white stars (汭). The white star means a voltage that is measured at the TDR input port per time unit. Thus, the forward model must estimate the values of the white stars.

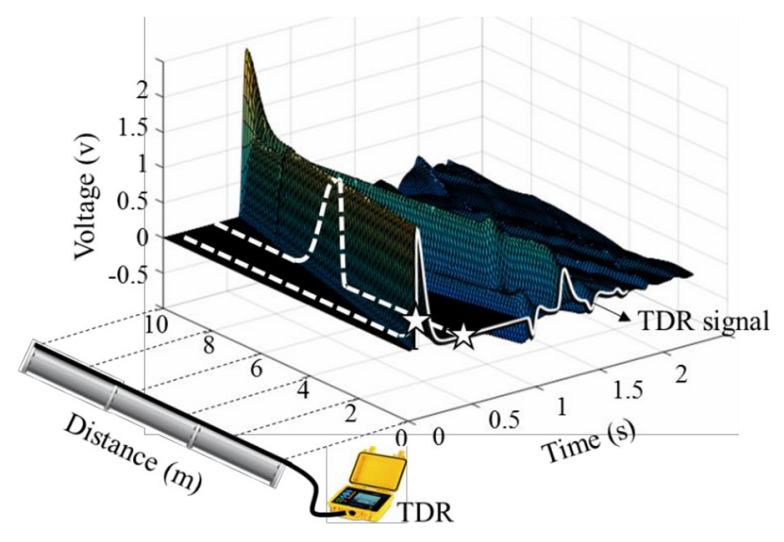

Figure 13. Voltage distribution on the transmission line in the time domain (white line: TDR signal; white dotted line: modeled signal based on RLCG) pipeline is 40 days which is reasonable tobe performed in the allotted construction period.

\subsection{Discussion}

In this section, the validated S-parameter based forward model is discussed in two points of view. First topic is physical reasons why the S-parameter based forward model is better in computational efficiency than the existing RLCG based forward model. Second topic is how well the proposed forward model is incorporated with the existing leak detection framework.

The physical advantages of S-parameter based forward model is as follows in comparison with previous RLCG based forward model. The TDR signal is a vector of sequent voltages that are measured at the TDR instrument. As shown in Fig. 13, the TDR signal is a white line that is composed of

The previous RLCG forward model, based on FDTD, requires calculating the all voltages (- - -, white dotted line) on the attached transmission line to obtain the one voltage ( 2 , white star) per time unit. However, the S-parameter based forward model does not need to calculate the all voltages. The S-parameter based model obtains the signal which is consisted of voltages in the frequency domain at the input port of the TDR equipment. Then, by performing IFFT, the frequency domain signal can be transformed to the time domain signal which is same as the white solid line. In other words, the S-parameter based model does not need to consider and calculate the white dotted lines. In this sense, the S-parameter based model is a more computationally economical method than the RLCG based model.

In regard to second topic, the sample data set, which is made by the S-parameter based forward model, is used in the existing leak detection framework to verify compatibility between the proposed forward model and the existing inverse model. And then the given leak condition as input data is a multiple-leak situation that has three leaks in a 10 meter pipeline. The locations of the leaks are at 5.5 meters, six 


\begin{tabular}{c|c|c|c|c|c|c}
\hline $\begin{array}{c}\text { Length of } \\
\text { pipeline }\end{array}$ & $\boldsymbol{t}_{\text {sample }}$ & $\mathbf{1 k m}$ & $\mathbf{5 k m}$ & $\mathbf{1 0 k m}$ & $\mathbf{2 0 k m}$ & $\mathbf{3 0 k m}$ \\
\hline$N_{\text {detector }}$ & - & 99 & 499 & 999 & 1,999 & 2,999 \\
\hline$N_{\text {sample data }}$ & 1 & 161,800 & $20,709,000$ & $166,168,000$ & $1,331,336,000$ & $4,495,504,000$ \\
\hline $\begin{array}{c}T_{\text {tot }} \text { of } \\
\text { S-para. model }[\mathrm{h}]\end{array}$ & $0.00263 \mathrm{~s}$ & 0.12 & 15.22 & $\begin{array}{c}122.15 \\
(5.09 \text { days })\end{array}$ & $\begin{array}{c}978.63 \\
(40.78 \text { days })\end{array}$ & $\begin{array}{c}3,304.53 \\
(137.69 \text { days })\end{array}$ \\
\hline $\begin{array}{c}T_{\text {tot }} \text { of } \\
\text { RLCG model }[\mathrm{h}]\end{array}$ & $3.81377 \mathrm{~s}$ & $\begin{array}{c}171.41 \\
(7.14 \text { days })\end{array}$ & $\begin{array}{c}21,938.73 \\
(2.5 \text { years })\end{array}$ & $\begin{array}{c}176,035.30 \\
(20.1 \text { years })\end{array}$ & $\begin{array}{c}1,420,392.95 \\
(161.0 \text { years })\end{array}$ & $\begin{array}{c}4,762,454.52 \\
(543.7 \text { years })\end{array}$ \\
\hline
\end{tabular}

Table 6 . The $T_{\text {tot }}$ in the field with three maximum detectable leaks

meters, and eight meters. As shown in Fig. 14 (a), the measured TDR signal is not explicit and thus cannot be interpreted by visual inspection. However, the location of the leaks can be stochastically found through using the Bayesian inference and the built sample data set, as shown in Fig. (b), which shows the marginal PDFs of each parameter around the location of the leaks. Therefore, S-parameter model is well compatibility in the framework of the existing modelbased leak detection system.

\section{CONCLUSiON}

A novel TDR-based leak detection system using an Sparameter forward model has been presented in this paper. The application of S-parameters improves the computational efficiency of the forward model and shortens the $T_{\text {sample }}$ of the sample data set needed for Bayesian inference. The Bayesian inference based inverse model can stochastically detect the location of leaks from an inexplicit TDR signal that includes noise and overlapped reflection. Moreover, to demonstrate the performance of the suggested leak detection system, laboratory experiments were conducted using a sample pipeline, leakage detectors, transmission lines, and TDR instrument. To simulate a long-distance pipeline, the length of each pipe unit was intentionally controlled at various lengths. Through the case study, the accuracy of the proposed

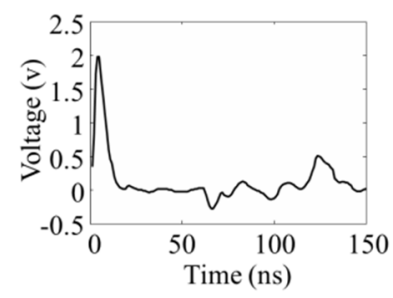

(a)

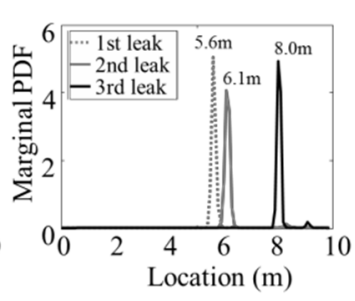

(b)
Figure 14. Bayesian inference for finding the location of leaks: (a) measured TDR signal, (b) location of leaks
S-parameter based forward model was validated by measures. The $t_{\text {sample }}$ was also obtained by averaging the results of different leak scenarios. Using the $t_{\text {sample }}$, the $T_{\text {tot }}$ including different lengths of pipeline and a varying number of maximum detectable leaks can be estimated.

As a result of this research, it is significantly meaningful that a multiple-leak detecting technique can be applied to a long water distribution system. Some people may think that a conventional TDR-based leak detection method is sufficient to detect pipeline leaks because the possibility of multiple leaks occurring simultaneously in any given pipeline is low. In addition, if it is easy to access the location of a particular leak, maintenance can be quickly performed as soon as a leak is detected and before another leak occurs. However, in actual pipeline applications if other leaks occur before an existing leak is repaired the usefulness of the conventional TDRbased method is limited. In addition, it can be difficult to perform quick maintenance on pipelines due to difficult accessibility (e.g., pipelines installed underwater or in desert or alpine regions). Moreover, the crustal movement of an area with installed pipelines, such as an earthquake, uplift of strata, ground sinking, or various external shocks, can force a change in the geometry of a long- distance pipeline. These phenomena may cause misalignment of a pipeline and generate multiple leaks at the flanges. Even though the nontrivial procedure of building a training data set is required for use of our proposed system, the suggested multiple-leak detection system offers significant long-term advantages, particularly in situations involving long-distance pipelines.

In terms of return on investment (ROI), the economic feasibility of the proposed detection system is superior to any existing method, including LNC, GPR, and PMA methods. While the TDR installation of this leak detection system, in terms of investment, could be regarded as an additional cost, the TDR installation, in terms of return, should not be regarded as an additional cost, but rather as an investment that will pay back in economic profits. In this regard, the installation of TDR system should be analyzed from the perspective of its long-term cost savings. First, in terms of 
operational cost reduction, the suggested method doesn't require surveyors and equipment to be dispatched for detecting leaks, unlike existing detection methods, because the proposed system can remotely monitor a wide area in real time. This advantage could derive benefits such as substantial labor cost savings. Second, in terms of cost avoidance, the price of the TDR instrument installed in the leak detection system is cheaper than it would be for electronic monitoring because the latter requires the TDR instrument with high resolution. In contrast, our suggested detection system requires only the TDR with moderate- resolution, which is able to accurately identify the distance between the detectors. In addition, our more robust system can also prevent the occurrence of costs related to economic, social, or environmental issues caused by being continuously unaware of leaks. Third, in terms of revenue growth, the net profit of the water industry is expected to gradually increase because of the reduction of non-revenue water (NRW) losses through more rapid maintenance to fix leaks.

If the economic efficiency of installation of the system is demonstrated in the long term, this leak detection system is also expected to be useful for sewer lines and wastewater pipelines. Generally, a leak of a sewer line or wastewater pipeline system is not related to an economic cost in the short term, so the effort put toward preventing leaks in these systems is relatively less than that observed for water distribution systems. However, leakage of sewage and wastewater may cause various environmental, economic, and social problems. First, the leakage causes soil and underground water contamination. It negatively impacts human health through agricultural products and can contaminate drinking water. Second, the contamination of soil and underground water leads to considerable remediation costs in the long term. Finally, the continuous leakage of wastewater pipelines can cause disasters such as sinkholes, in which a hole is made by the collapse of the ground surface as a result of a leaking pipeline below ground (Gutiérrez, Galve, Guerrero, Lucha,, Cendrero, \& Remondo, 2007). This situation may result in great a catastrophe causing many casualties. Thus, applying the proposed TDR system to sewer lines and wastewater pipelines could prevent these problems in the long term.

Future work related to this research will be expanded to examine the network structure of pipelines because this research is only applicable to a single pipeline. As a result, this method would need multiple TDR instrument stations to cover a networked pipeline structure. In the case of coaxial cable, Xiaolong Zhang has examined the failure diagnosis technique of a cable network using a TDR-based system using a modeling splitter and tap (Zhang, Zhang, \& Liu, 2013). However, to be robust to noise and improve interpretability of the TDR signal for multiple leaks, a Bayesian inference based network detection technique must be developed. Thus, to reduce the amount of required equipment and cost, the authors will seek to develop a method that efficiently detects leaks in a networked water distribution system.

\section{ACKNOWLEDGEMENTS}

This work was supported by the Mid-Career Researcher Program through the National Research Foundation of Korea (NRF) grant funded by the Ministry of Science ICT and Future Planning (MSIP) (2013R1A2A2A01068627). This work was also supported by a grant from the Institute of Advanced Machinery and Design at Seoul National University (SNU-IAMD).

\section{REFERENCES}

Hunaidi, O., Chu, W., Wang, A., \& Guan, W. (2000). Detecting leaks. JOURNAL AWWA, vol. 92, pp. 82-94.

Cheong, L. C.. (1991). Unaccounted for water and the economics of leak detection. Proc. International Water Supply Congress and Exhibition, Copenhagen.

Thornton, J., Sturm, R., \& Kunkel, G. (2008). Water loss control: McGraw Hill Professional.

Moe, C. L., \& Rheingans, R. D. (2006). Global challenges in water, sanitation and health. Journal of water and health, vol. 4, p. 41.

CNT(The Center for Neighborhood Technology) (2013). The Case for fixing the Leaks: Protecting people and saving while supporting economic growth in the Great Lakes region. http://www.cnt.org/publications/the-casefor- fixing-the-leaks-protecting-people-and-savingwater-while-supporting.

Gao, Y., Brennan, M., Joseph, P., Muggleton, J., \& Hunaidi, O. (2004). A model of the correlation function of leak noise in buried plastic pipes. Journal of Sound and Vibration, vol. 277, pp. 133-148.

O'Brien, E., Murray, T., \& McDonald, A. (2003). Detecting leaks from water pipes at a test facility using groundpenetrating radar. Pumps, Electromechanical Devices and Systems Applied to Urban Water Management, vol. 1, p. 395.

Demirci, S., Yigit, E., Eskidemir, I. H., \& Ozdemir, C. (2012). Ground penetrating radar imaging of water leaks from buried pipes based on back- projection method. NDT \& E International, vol. 47, pp. 35-42.

Costello, S., Chapman, D., Rogers, C., \& Metje, N. (2007). Underground asset location and condition assessment technologies. Tunnelling and Underground Space Technology, vol. 22, pp. 524- 542.

McNulty, J. (2001). An acoustic-based system for detecting, locating and sizing leaks in water pipelines. Proceedings of the 4th International Conference on Water Pipeline Systems: Managing Pipeline Assets in an Evolving Market. York, UK.

Vítkovský, J. P., Lambert, M. F., Simpson, A. R., \& Liggett, J. A. (2007). Experimental observation and analysis of inverse transients for pipeline leak detection. Journal of 
Water Resources Planning and Management, vol. 133, pp. 519-530.

Ghazali, M., Staszewski, W. J., Shucksmith, J., Boxall, J. B., \& Beck, S. B. (2010). Instantaneous phase and frequency for the detection of leaks and features in a pipeline system. Structural Health Monitoring.

Covas, D., Ramos, H., \& De Almeida, A. B. (2005). Standing wave difference method for leak detection in pipeline systems. Journal of Hydraulic Engineering, vol. 131, pp. 1106-1116.

Brunone, B. (1999). Transient test-based technique for leak detection in outfall pipes. Journal of water resources planning and management, vol. 125, pp. 302-306.

Puust, R., Kapelan, Z., Savic, D., \& Koppel, T. (2010). A review of methods for leakage management in pipe networks. Urban Water Journal, vol. 7, pp. 25-45.

Kim, Y., Suh, J., Cho, J., Singh, S., \& Seo, J. (2015). Development of Real-Time Pipeline Management System for Prevention of Accidents. International Journal of Control and Automation, vol. 8, pp. 211- 226.

Cataldo, A., Cannazza, G., De Benedetto, E., \& Giaquinto, N. (2012). A TDR-based system for the localization of leaks in newly installed, underground pipes made of any material. Measurement Science \& Technology, vol. 23, p. 9.

Cataldo, A., Cannazza, G., De Benedetto, E., \& Giaquinto, N. (2012). A new method for detecting leaks in underground water pipelines. Sensors Journal, IEEE, vol. 12, pp. 1660-1667.

Cataldo, A., Cannazza, G., De Benedetto, E., \& Giaquinto, N. (2012). Experimental validation of a TDR-based system for measuring leak distances in buried metal pipes. Progress in Electromagnetics Research- Pier, vol. 132, pp. 71-90.

Kim, T., Woo, S., Youn, B. \& Huh, Y. (2015), TDR-based Pipe Leakage Detection and Location using Bayesian Inference. Prognostics and Health Management (PHM), 2015 IEEE Conference on, pp. 1-5.

O'Connor, K. M. \& Dowding, C. H. (1999), Geomeasurements by pulsing TDR cables and probes: CRC Press.

Yang, X., Choi, M.-S., Lee, S.-J., Ten, C.-W., \& Lim, S. -I. (2008). Fault location for underground power cable using distributed parameter approach. Power Systems, IEEE Transactions on, vol. 23, pp. 1809- 1816.

Kwon, D., Azarian, M. H., \& Pecht, M. (2009). Early Detection of Interconnect Degradation by Continuous Monitoring of RF Impedance. Ieee Transactions on Device and Materials Reliability, vol. 9, pp. 296-304.

Yu, X., Zhang, B., Tao, J., \& Yu, X. (2013). A new timedomain reflectometry bridge scour sensor. Structural Health Monitoring, vol. 12, pp. 99-113.

Yu, X. \& Yu, X. (2009). Time domain reflectometry automatic bridge scour measurement system: principles and potentials. Structural Health Monitoring, vol. 8, pp. 463-476.
Calamita, G., Brocca, L., Perrone, A., Piscitelli, S., Lapenna, V., \& Melone, F., et al (2012). Electrical resistivity and TDR methods for soil moisture estimation in central Italy test-sites. Journal of Hydrology, vol. 454, pp. 101-112.

Ledieu, J., De Ridder, P., De Clerck, P., \& Dautrebande, S. (1986). A method of measuring soil moisture by timedomain reflectometry. Journal of Hydrology, vol. 88, pp. 319-328.

Di Sante, R. (2005). Time domain reflectometry-based liquid level sensor. Review of Scientific Instruments, vol. 76, p. 5.

Wang, P. F., Youn, B. D., Xi, Z. M., \& Kloess, A. (2009). Bayesian Reliability Analysis With Evolving, Insufficient, and Subjective Data Sets. Journal of Mechanical Design, vol. 131.

Wang. P. F., Youn, B. D., \& Hu, C. (2012). A generic probabilistic framework for structural health prognostics and uncertainty management. Mechanical Systems and Signal Processing, vol. 28, pp. 622-637.

Reinhold, L., \& Pavel, B. (2000). RF circuit design: theory and applications : Prentice Hall Upper Saddle River.

Schuet, S., Timucin, D., \& Wheeler, K. (2011). A ModelBased Probabilistic Inversion Framework for Characterizing Wire Fault Detection Using TDR. Ieee Transactions on Instrumentation and Measurement, vol. 60, pp. 1654-1663.

Miller, S. J. (2006). The method of least squares. Mathematics Department Brown University, pp. 1- 7.

Twisk, D., Spit, H., Beebe, M., \& Depinet, P. (2007). Effect of Dummy Repeatability on Numerical Model Accuracy. SAE Technical Paper 0148-7191.

Gutiérrez, F., Galve, J., Guerrero, J., Lucha, P., Cendrero, A., \& Remondo, J., et al. (2007). The origin, typology, spatial distribution and detrimental effects of the sinkholes developed in the alluvial evaporite karst of the Ebro River valley downstream of Zaragoza city (NE Spain). Earth Surface Processes and Landforms, vol. 32, pp. 912-928.

Zhang, X., Zhang, M., \& Liu, D. (2013). Practicable model of coaxial cable channel with splitter and tap via statetransition matrix. Measurement, vol. 46, pp. 1190-1199. 